\title{
Renal Bed
}

National Cancer Institute

\section{Source}

National Cancer Institute. Renal Bed. NCI Thesaurus. Code C131138.

The cavity evident following excision of a kidney. The renal bed is a potential site of recurrent disease post-nephrectomy. 